\title{
Tomographic diagnostic of the hydrogen beam from a negative ion source
}

\author{
M. Agostini, M. Brombin, ${ }^{*}$ G. Serianni, and R. Pasqualotto \\ Consorzio RFX, Associazione EURATOM-ENEA sulla fusione, Corso Stati Uniti 4, Padova, Italy
}

(Received 1 August 2011; published 4 October 2011)

\begin{abstract}
In this paper the tomographic diagnostic developed to characterize the 2D density distribution of a particle beam from a negative ion source is described. In particular, the reliability of this diagnostic has been tested by considering the geometry of the source for the production of ions of deuterium extracted from an rf plasma (SPIDER). SPIDER is a low energy prototype negative ion source for the international thermonuclear experimental reactor (ITER) neutral beam injector, aimed at demonstrating the capability to create and extract a current of $D^{-}\left(H^{-}\right)$ions up to $50 \mathrm{~A}(60 \mathrm{~A})$ accelerated at $100 \mathrm{kV}$. The ions are extracted over a wide surface $\left(1.52 \times 0.56 \mathrm{~m}^{2}\right)$ with a uniform plasma density which is prescribed to remain within $10 \%$ of the mean value. The main target of the tomographic diagnostic is the measurement of the beam uniformity with sufficient spatial resolution and of its evolution throughout the pulse duration. To reach this target, a tomographic algorithm based on the simultaneous algebraic reconstruction technique is developed and the geometry of the lines of sight is optimized so as to cover the whole area of the beam. Phantoms that reproduce different experimental beam configurations are simulated and reconstructed, and the role of the noise in the signals is studied. The simulated phantoms are correctly reconstructed and their two-dimensional spatial nonuniformity is correctly estimated, up to a noise level of $10 \%$ with respect to the signal.
\end{abstract}

DOI: 10.1103/PhysRevSTAB.14.102801

PACS numbers: 52.25.Tx

\section{INTRODUCTION}

In this paper the feasibility of a visible tomography diagnostic of a beam from negative ion source is demonstrated through the description of a conceptual design, the development of an inversion algorithm, and the simulation of the expected performances. The system described herein is compatible with the mechanical constraints, i.e. port location and beam dimensions, of the source for the production of ions of deuterium extracted from an rf plasma (SPIDER) negative ion source which will operate with hydrogen or deuterium at $100 \mathrm{keV}$ with an extracted current density around $300 \mathrm{Am}^{-2}$ for pulses up to $3600 \mathrm{~s} \mathrm{[1].}$ Spectroscopy observing the radiation emitted by the particles is widely and successfully used to characterize the average properties both of the plasma sources and of the extracted beams (see, for example, [2-4] and references cited there). Tomography aims at measuring the emission or the absorption of radiation by an object, using a large number of lines of sight (LoSs). The final outcome is the reconstruction of a $2 \mathrm{D}$ or $3 \mathrm{D}$ profile of emission or absorption. It is a technique routinely used in medicine and invented by Nobel laureates Cormack and Hounsfield in the 1970s (see, for example, [5] and references therein). The emission tomography measures the brightness $f$ of an emissive source along a set of lines of sight, $l_{j}$ :

\footnotetext{
*matteo.brombin@igi.cnr.it
}

Published by the American Physical Society under the terms of the Creative Commons Attribution 3.0 License. Further distribution of this work must maintain attribution to the author(s) and the published article's title, journal citation, and DOI.

$$
f_{j}=\int_{l_{j}} \epsilon(x, y) d x d y,
$$

where the integral is evaluated along the $j$ th line of sight. If the number of LoSs is sufficient, it is possible to obtain the function $\epsilon(x, y)$ from the brightness measurements. So the problem to be solved is an inversion problem. For the particular tomographic configuration considered in the paper, $\epsilon(x, y)$ is the emissivity of the $H^{-}$beam and this is the quantity that has to be reconstructed. The diagnostic analyzes the $H_{\alpha}\left(D_{\alpha}\right)$ light emitted along the LoSs on a plane perpendicular to the beam.

\section{Visible tomography in SPIDER: Motivation and requirements}

The main goal of tomographic diagnostic investigated in this paper is the assessment of the uniformity of the beam. Considering the particular case of SPIDER, a beam homogeneity in terms of extracted current density of $\pm 10 \%$ is required [6]. So the purpose of this work is to demonstrate the capability of the proposed diagnostic to reconstruct a nonuniform beam profile with a difference between the tomographic reconstruction and the real emissivity of the beam sufficiently lower than the actual deviation from uniformity. The following requirements are assumed for the tomographic diagnostic: (i) measurement of LoS-integrated $H_{\alpha}\left(D_{\alpha}\right)$ radiation; (ii) suitable number and arrangement of LoSs, so that a good spatial resolution is obtained from the inversion; (iii) time resolution better than $10 \mathrm{~Hz}$.

The application of tomography to a neutral or ion beam can actually give more information than just the level of 
uniformity, which is achievable by the line-integrated emissivity over few LoSs, without the use of inversion techniques, as performed for example in the MANITU experiment [2].

Maximizing the spatial uniformity of the accelerated beam extracted from negative ion sources has been found one of the most difficult problems to solve [7-9]: since the causes are not yet completely understood, this problem is not completely solved in the existing beams. So, a complete reconstruction of the beam emissivity in two dimensions obtained with the proposed tomography can go beyond the simple detection of the lack of uniformity of the beam, giving information about its causes and suggesting possible solutions. The development of a visible tomography for diagnosing a beam is not a novelty, but, to the authors' knowledge, it has never been used to detect small irregularities of the beam [i.e. $\pm 10 \%$ as required for SPIDER and International Thermonuclear Experimental Reactor (ITER) neutral beam] and it has never been used for beams extracted from negative ion sources. The first tomographic reconstruction applied to a high power beam extracted from a positive ion source dates back to the 1980s: a visible tomographic diagnostic was developed for the JET neutral beam $[10,11]$. After that, tomography is discussed for the KSTAR NBI [12], and a general study of the diagnostic can be found in [13].

When the beam is extracted and accelerated from a positive ion source, it is built up by many beamlets that are very small (with respect to the negative beams) and that merge very near to the grid, so the required spatial resolution is lower, while a beam extracted and accelerated from a negative ion source is composed by a pattern of small and well-separated beamlets whose identity can be recognized up to a distance of few meters. This behavior has been shown in the two largest negative ion beams operating nowadays: the NBI of LHD and JT60-U. In JT60-U and in the Kamaboko source it was shown that the single beamlets are well separated and visible at several meters (from 1 up to 3 ) downstream the ion source [7,14-16]. So a tomographic diagnostic for this type of beams should take into account this spatial resolution. Therefore a negative ion source has to be simulated and inverted as a matrix of $n$ Gaussians, $n$ being the number of beamlets: this spatial discretization of the beam has to be taken into account in the inversion algorithm.

The paper is organized as follows: in Sec. I an introduction to tomography and its use in accelerated beams is given; Sec. II is focused on the description of the geometry of SPIDER [1], a particular negative ion source on which all the designs and simulations further explained have been carried out. The layout of detectors and the description of the lines of sight is reported in this section. Then in Sec. III the simulated phantoms are presented: they reproduce different possible emissivity profiles that the tomography has to reconstruct. The method used for solving the inversion problem is discussed in Sec. IV. Some relevant phantoms and their tomographic inversions are presented and discussed in Sec. V, where also a sensitivity analysis is given. Section VI is dedicated to the conclusions.

\section{GEOMETRY DEFINITION}

The collection and detection system of the visible tomography system observes at the beam through a set of viewports arranged all around the vacuum vessel on a transverse section, as illustrated in Fig. 1, where the available ports are numbered from 1 to 15 . On each port a linear CCD or equivalent detector array measures the light emitted by fast particles of the beam via charge exchange with the background gas. In the present study each detector array collects the LoS-integrated optical signals of a fan of LoSs on a plane perpendicular to the beam. Observing the beam emissivity with a sufficiently large number of LoSs will allow a tomographic reconstruction of the two-dimensional beam emission function $\epsilon(x, y)$ which is proportional to the beam density, on the assumption that the density of the background gas is uniform across the observed plane. This is a critical condition for the diagnostic, especially for long beam pulse, but given the characteristics of the vacuum pumping system it is expected to be verified.

\section{A. Constraint description: Vacuum vessel and port location}

The model of the tomography geometry presented in Fig. 2 is helpful to highlight the mechanical constraints

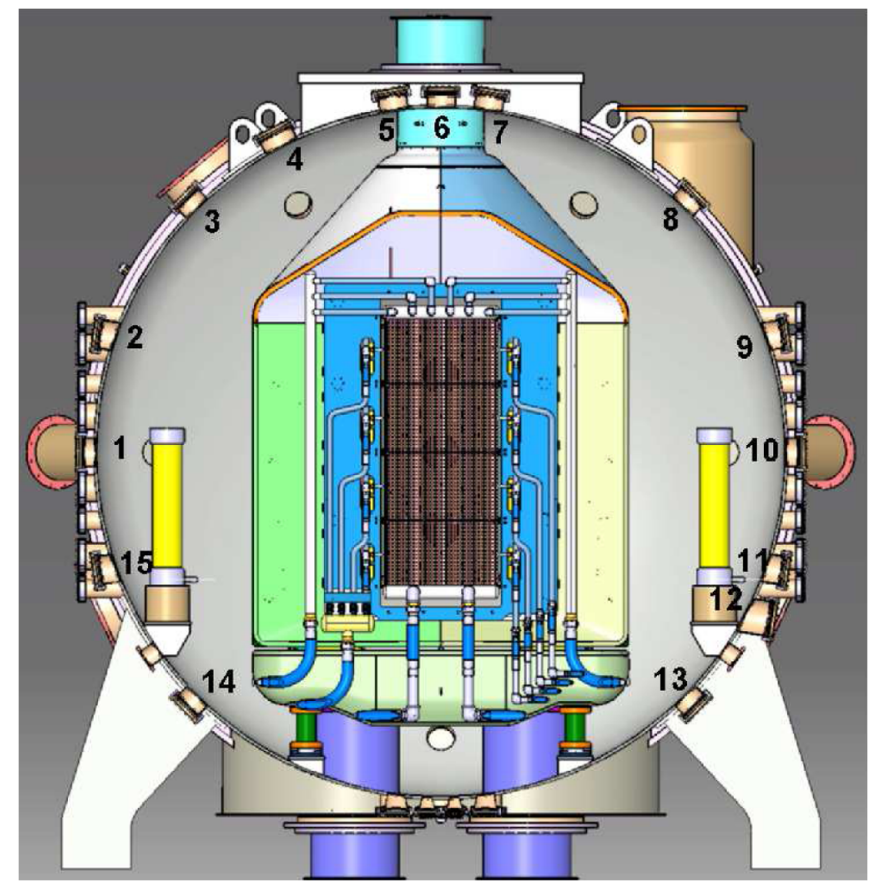

FIG. 1. SPIDER vacuum vessel cross section. The 15 ports used for the tomography diagnostic are numbered clockwise starting from the left equatorial one. 


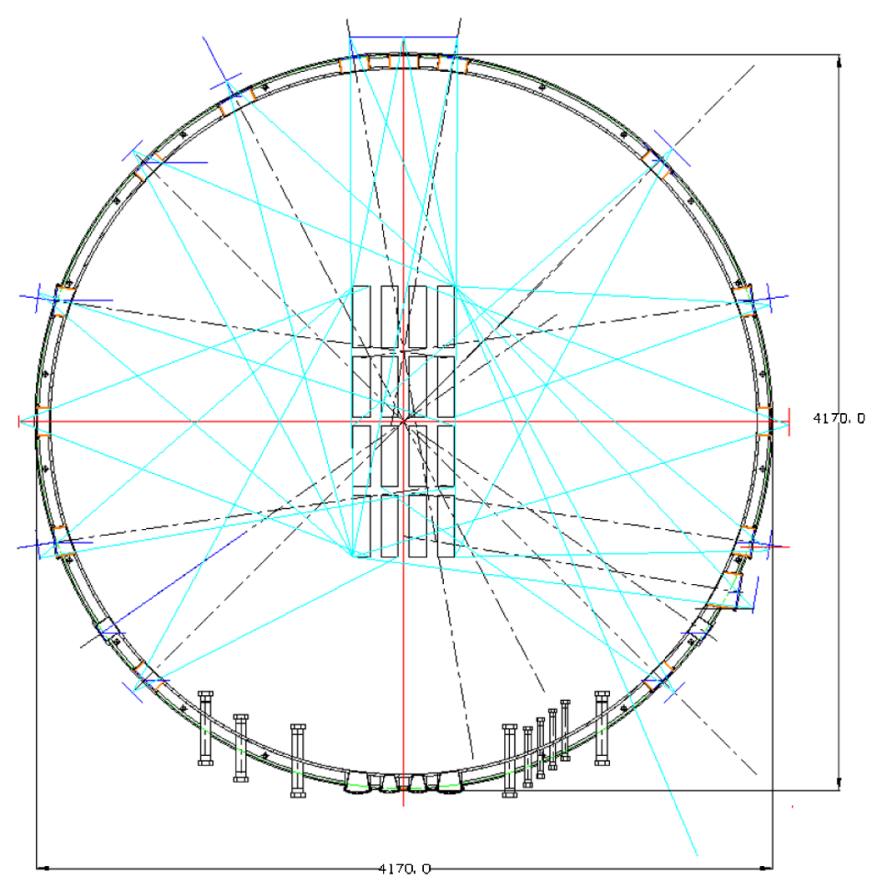

FIG. 2. Fan distribution for the best coverage of the beam cross section. The blue lines define the boundaries of each fan. The vertices are placed $100 \mathrm{~mm}$ far from the window of the corresponding viewport. The 16 rectangles in the center are the 16 beamlet groups that constitute the beam.

which have been taken into account to design the diagnostic. The big circle is the vessel with the 15 available observation portholes, and the internal rectangles represent the 16 beamlet groups, each made up of $5 \times 16$ beamlets. A full coverage of the beam section by means of an adequate number of LoSs has to be guaranteed. Moreover, any symmetric arrangement has to be avoided in order to approach as much as possible a complete coverage of all viewing directions over $360^{\circ}$, without any repetition which would not increase the information content. Since some portholes are symmetric, different optical axes for each port are adopted.

\section{B. Ports needed and ports available}

The number of projections $\left[f_{j}\right.$ of Eq. (1)] and, hence, of image sensors needed for a sufficiently good tomographic reconstruction of the emitting beam can be determined using the Fourier slice theorem, which relates the measured projection data to the two-dimensional Fourier transform of the object cross section. According to this theorem, for a fan with parallel LoSs, the Fourier transform of the projection along a line of sight is equal to the two-dimensional Fourier transform of the object along a line perpendicular to the LoSs [17]. The number of needed ports (projections) depends on the desired space resolution. An approximate formula can therefore be applied to a preliminary estimation of the minimum number of detectors providing a given space resolution. The number of ports has also to fulfill technical limitations, such as space limitations and/or design constraints. As a first approximation, the number of cameras needed to reconstruct the emitting region can be estimated through [10]

$$
N=\frac{\pi D}{\Delta s}
$$

where $\Delta s$ is the dimension of the minimum resolvable object, and $D$ the extension of the emitting plasma region. In the particular case described in this paper, $D=1 \mathrm{~m}$ is the vertical dimension of SPIDER beam, and $\Delta s=0.1 \mathrm{~m}$ is the required spatial resolution. This equation indicates that the number of projections needed for a complete reconstruction without noise to be $N \approx 30$. In SPIDER there are only 15 ports available, so about half of the needed projections. Moreover, the line-integrated signals are always affected by noise. However, there are mathematical methods, discussed in the following paragraphs, which allow the image reconstruction despite the limited amount of input data.

\section{General description}

The design of the fans for the visible tomography diagnostic is carried out by starting from the scheme of the SPIDER vessel cross section (see Fig. 2) where the portholes and the positions of the beamlet groups are shown; this is done by placing a camera in each available porthole and defining features of the LoSs of each fan. It is clear that portholes $3,8,13$, and 14 are symmetric and also portholes $2,9,11$, and 15 . This symmetry, as previously said, must be broken by a suitable variation of the orientation of the fans, to increase the amount of independent information. Figure 2 shows the distribution of the fans, each identified by the two external LoSs. In particular, Fig. 2 reports the final configuration of the fans which has been considered for the entire analysis here presented. It is the result of several iterations to find out the best combination of LoSs to reconstruct the emissivity profile. The optimization was carried out by minimizing the error of the reconstructed pattern [see Eq. (15)] with respect to a known input, i.e. phantom 8 presented in Sec. VA. In case of no noise in the experimental measurements, many configurations can correctly reconstruct the emissivity; therefore a finer optimization was carried out by comparing the reliability of these different solutions when an equal noise level is added to the measurements. In such a way, the solution proposed in this work is the one which provides the best inversion also in the case of noisy measurements. The coverage of the beam area by the LoSs of the tomographic system is shown in Fig. 3: each LoS is identified by its impact parameter $p$ (the distance from the center of the beam) normalized to half the vertical dimension of the beam $(b)$ and by its inclination angle $(f)$. Each point in the plane $(p, f)$ represents one LoS. 


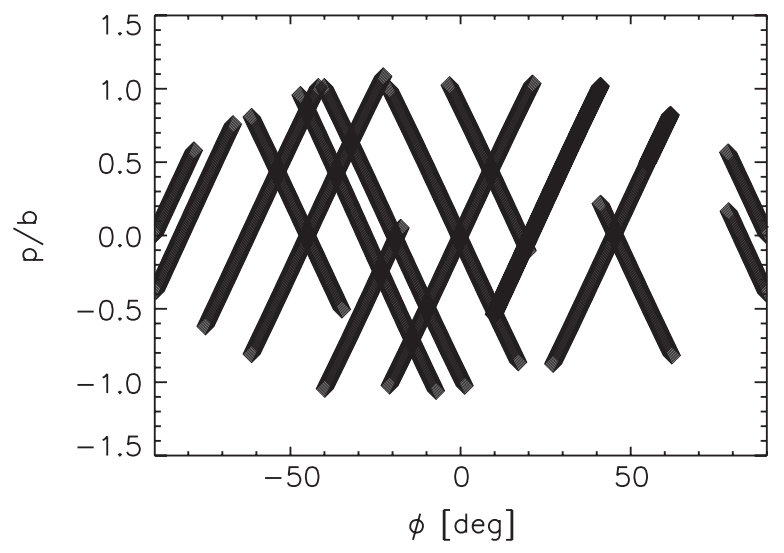

FIG. 3. Experimental coverage in the plane $(\phi, p)$ for the tomography diagnostic. $b$ is half the vertical dimension of the beam, $p$ is the impact parameter of the LoS, and $\phi$ is its angle.

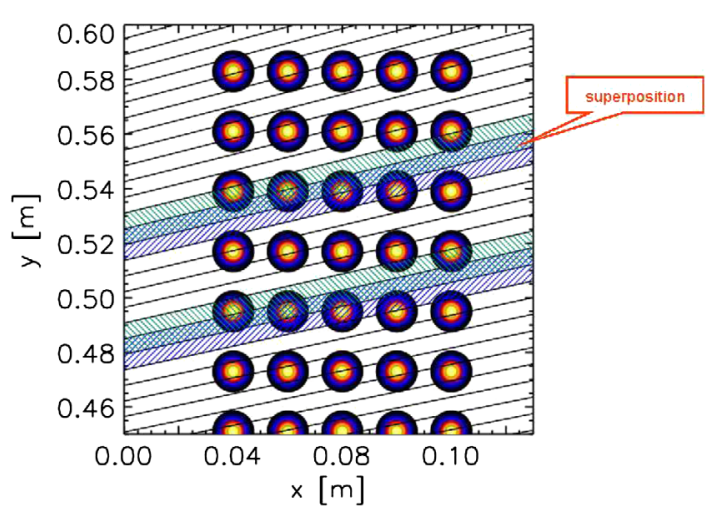

FIG. 4. Representation of lines of sight. The picture highlights two cases of superposition between two neighboring LoSs. The blue or green areas are LoSs. The region where two colors are superimposed is the area belonging to both contiguous LoS. Each LoS is half covered by the previous one and half by the next one. In the picture also the Gaussian beamlets are visible.

\section{Lines of sight}

All lines of sight have an angular aperture of $2.5 \mathrm{mrad}$, so they have a finite spatial width. This corresponds to a width of $10 \mathrm{~mm}$ at $2 \mathrm{~m}$, that is the average distance from a porthole to the beamlets. The LoSs overlap with each other and the number of LoSs for each fan has been calculated using the rule that half of each LoS is covered by the neighboring LoSs. Figure 4 shows the superposition between contiguous lines in a selected area of the observed plane. The total number of LoS in the proposed visible tomography design is 3127 , covering almost uniformly the beam (Fig. 2).

\section{PHANTOMS}

The phantom, i.e. the two-dimensional emissivity profile of the beam which has to be reconstructed by the visible tomography system, reproduces the beam pattern with well distinguishable beamlets. To this purpose, the emission profile of every single beamlet is schematized as a twodimensional Gaussian surface. Thus, the beam is simulated as an array of 1280 Gaussian beamlets:

$$
G_{g}(x, y)=A_{g} e^{\left[\left(x-x_{0 g}\right)^{2} / 2 \sigma_{x g}^{2}\right]-\left[\left(y-y_{0 g}\right)^{2} / 2 \sigma_{y g}^{2}\right]} .
$$

Every beamlet has five free parameters that can be varied to generate different phantoms and simulate different physical situations: the amplitude $A_{g}$, the position along $x$ and $y$ directions $\left(x_{0 g}, y_{0 g}\right)$, and the width along $x$ and $y$ $\left(\sigma_{x g}, \sigma_{y g}\right)$. Within each beamlet group, the beamlets are spaced by $20 \mathrm{~mm}$ in the horizontal direction and by $22 \mathrm{~mm}$ in the vertical direction; facing external beamlets of neighboring beamlet groups are spaced by $80 \mathrm{~mm}$ in the horizontal direction and by $60 \mathrm{~mm}$ in the vertical direction.

\section{Line integral evaluation}

Once the phantom is generated, the line-integrated signal of the emissivity along the $j$ th $\operatorname{LoS}$ is calculated $\left(I_{j}\right)$. Since the LoSs have a finite width, to simulate the real situation the line-integrated signal is calculated by integrating the emissivity of all Gaussian beamlets which are inside the two straight lines that define a LoS. Defining the two straight lines as

$$
y=a_{1}+b_{1} x \quad y=a_{2}+b_{2} x,
$$

the line-integrated signal is

$$
I=f_{1}-f_{2}
$$

where $f_{1}$ is the integral of all the emitting Gaussians under line 1 and $f_{2}$ is the integral of all the emitting Gaussians under line 2 . Hence, the difference between the two integrals is the light collected by each 2D LoS (i.e. the light between the lines 1 and 2). Explicitly,

$$
\begin{aligned}
f_{1}= & \frac{\pi}{2} \sum_{g} A_{g} \sigma_{g}^{2} \\
& \times\left[1+\operatorname{Erf}\left(\frac{\left(y_{c}-y_{0 g}\right) \cos \left(\varphi_{1}\right)-\left(x_{c}-x_{0 g}\right) \sin \left(\varphi_{1}\right)}{\sqrt{2} \sigma_{g}}\right)\right] \\
& \cdot \operatorname{Erfc}\left(\frac{\left(x_{c}-x_{0 g}\right) \cos \left(\varphi_{1}\right)+\left(y_{c}-y_{0 g}\right) \sin \left(\varphi_{1}\right)}{\sqrt{2} \sigma_{g}}\right)
\end{aligned}
$$

and $f_{2}$ is an analogous quantity obtained by replacing the subscript 1 with 2 . All the symbols with the subscript $g$ refer to the Gaussian beamlets, $x_{c}$ and $y_{c}$ are the coordinates of the vertex of the fan, and $\varphi_{1}$ and $\varphi_{2}$ the angles of the two lines that define the LoS, defined as

$$
\varphi_{1}=\arctan \left(b_{1}\right) \quad \varphi_{2}=\arctan \left(b_{2}\right) .
$$

The functions $\operatorname{Erf}$ and $\operatorname{Erfc}$ are defined in the usual way as

$$
\operatorname{Erf}(z)=\frac{2}{\sqrt{\pi}} \int_{0}^{z} e^{-t^{2}} d t \quad \operatorname{Erfc}(z)=1-\operatorname{Erf}(z) .
$$


In order to simplify the mathematics, in the formula $\sigma_{x}=\sigma_{y}$ is assumed (without this assumption the integration of the Gaussian emissivity inside a LoS should be calculated numerically). Once the phantom has been defined, using Eq. (6) it is possible to evaluate all the line-integrated signals simulating the experimental signals collected by the camera, which will be used as input for the tomographic inversion. In a realistic situation, the experimental signals would be affected by noise, at least from the $\mathrm{CCD}$. This is simulated by adding to each line-integrated signals a random noise proportional to its value and with uniform distribution. Different noise levels have been considered, with the rms of the noise/signal from $0 \%$ (no noise) up to $20 \%$. For the CCD considered so far as candidate detector for the tomographic diagnostic, the noise due to the detector is expected to be $<5 \%$, as experimentally tested.

\section{INVERSION TECHNIQUE}

There are different numerical methods which can be developed and used to make the tomographic inversion of a 2D emissivity structure by measuring line-integrated signals, and the choice depends on different aspects of the problem: structure of the image, a priori knowledge of the emission function, mathematically overdetermined or underdetermined system, and noise level [18]. For tomography in SPIDER, there is good a priori knowledge of the expected emissivity of the beam, in particular its geometry is known: the beam is composed of 1280 beamlets arranged in a well-defined array resembling the holes on the source grid. In a previous work [19], the problem was tackled using the pixel method and solving the overdetermined linear problem with the singular value decomposition (SVD) technique. With that method it has been possible to make a tomographic reconstruction of the phantoms with noisy data obtaining a space resolution of one beamlet group. In order to obtain a better spatial resolution and also to reduce the error in the reconstructed image, even with noisy line-integrated signals, the number of pixels was increased, a different inversion technique instead of the SVD is used, and also a different definition of pixel is adopted.

\section{A. The method}

Using the pixel method, the unknowns are the emissivities of each pixel $i$ of the image, namely $\epsilon_{i}$. So, being $I_{j}$ the line-integrated signal of the line $j$, the tomographic problem can be written in matrix form:

$$
I_{j}=\sum_{i} \epsilon_{i} a_{i, j}
$$

where $a_{i j}$ is the fraction of area of pixel $i$ observed by line $j$. In this form, the emissivity of each pixel is formally obtained by inverting the matrix $a$ :

$$
\epsilon=\mathbf{I} a^{-1}
$$

The problem of tomographic inversion is ill posed, since the matrix $a$ is sparse, i.e. it contains a large number of zeros. The more measurements are available, the smaller the number of zeros in the matrix for a given pattern of pixels. So, the nature of the inversion algorithm depends on the characteristics of the matrix $a$, and the matrix depends on the geometry of LoSs and on the definition of the pixels. Before describing the inversion technique in more detail, the new definition of pixel is introduced and discussed in the next paragraph.

\section{B. Matrix $a_{i j}$ : Description}

In order to obtain a good tomographic reconstruction of an image, all the a priori knowledge should be used. In the case of SPIDER, the beam is made up of 1280 beamlets whose emissivities have a Gaussian shape, arranged into 16 rectangular shaped beamlet groups. For the reconstruction, the beam is divided into 32 rectangular pixels of equal size, as shown in Fig. 5, and each of them is made of $40(5 \times 8)$ Gaussians. These Gaussians are all centered in their nominal positions and they have all the same width (the nominal one $\sigma_{x g}=\sigma_{y g}=3 \mathrm{~mm}$ ). The only free parameters are the amplitudes $A_{g}$, but with the constraint that inside each pixel all the Gaussians have the same amplitude. So a pixel is not simply a rectangular region: it is composed by 40 identical Gaussians with the same amplitude. Using this definition of pixel, the components of the matrix $a$ are evaluated as

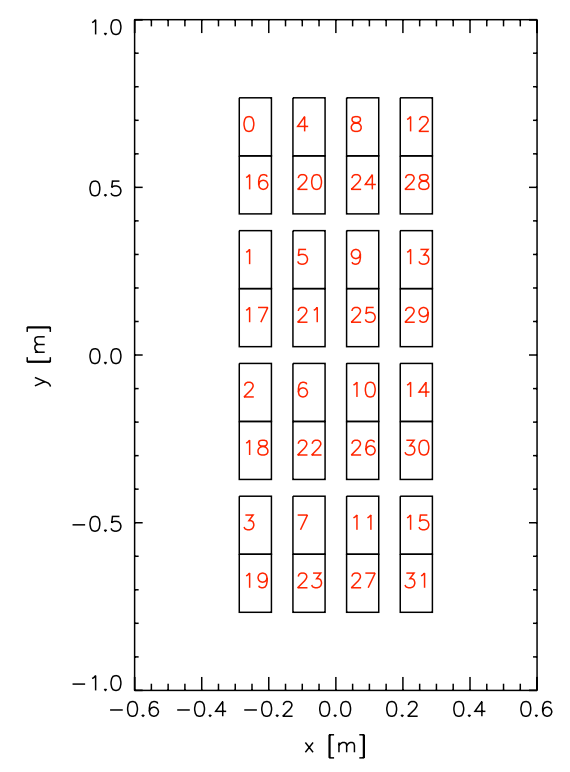

FIG. 5. Scheme of the pixels used for the tomographic reconstruction. The configuration with 32 pixels is shown (each beamlet group is divided into two pixels). 


$$
a_{i j}=\int_{l_{j}} \sum_{g \in i} G_{g} \quad \text { with } A_{g}=1
$$

So the component $a_{i j}$ of the matrix is the integral of the emissivity of all Gaussians $G_{g}$ with amplitude $A_{g}=1$ belonging to the pixel $i$ that are inside the two-dimensional line of sight (see Fig. 4) $l_{j}$. Thus, after evaluating the matrix $a$ and upon having the line-integrated signals I, the emission of each pixel (i.e. the amplitude of all the Gaussians belonging to that pixel) is computed according to Eq. (10).

\section{SART method}

The method used to solve this linear system of equations is described in the following. In order to solve the inverse problem of the tomography in SPIDER, two methods have been considered so far: the SVD $[17,20]$ and the algebraic reconstruction technique (ART) [18,21-23], in particular in its modified form called simultaneous algebraic reconstruction technique (SART) [24]. The SART technique has been preferred to the SVD because it gives better results when noisy data are used, as described also in the cited references. In fact, for the specific application considered in this paper, SVD and SART are equivalent when no noise is added to the line-integrated signals, while SART gives more accurate reconstruction when noise is added. SART is an iterative technique which allows solving the linear system of Eq. (10) via an iterative "error-correcting" procedure, which can be written as

$$
\epsilon_{i}^{(k+1)}=\epsilon_{i}^{(k)}+\frac{\sum_{j}\left[a_{i j} \frac{\mathbf{I}_{j}-\mathbf{a}_{j} \cdot \epsilon^{(k)}}{\sum_{i} a_{i j}}\right]}{\sum_{j} a_{i j}},
$$

where $a_{j}$ denotes the $j$ th column vector of matrix $a$, and $\epsilon^{(k)}$ is the emissivity of pixel $i$ after $k$ iterations. The initial estimate $\epsilon^{(0)}$ is set to 0 . The second term of Eq. (12) is the correction term, which is averaged over all LoSs $(j)$. Since the expected emissivity of each pixel is positive, this constraint is enforced in each iteration by setting to zero the coefficients that are less than zero after an iterative step.

\section{INVERSION RESULTS}

In this section the tomographic inversion is applied to the phantoms introduced in Sec. III, and the results are discussed.

\section{A. Nonuniform phantom}

This phantom simulates a beam which is not uniform in both $x$ and $y$ directions, with the emission which varies linearly in the two directions. The amplitudes of the 1280 Gaussian beamlets are defined as

$$
A_{g}(x, y)=A_{0, g}\left(1+\delta x \frac{x_{0 g}}{\Delta x}+\delta y \frac{y_{0 g}}{\Delta y}\right) .
$$
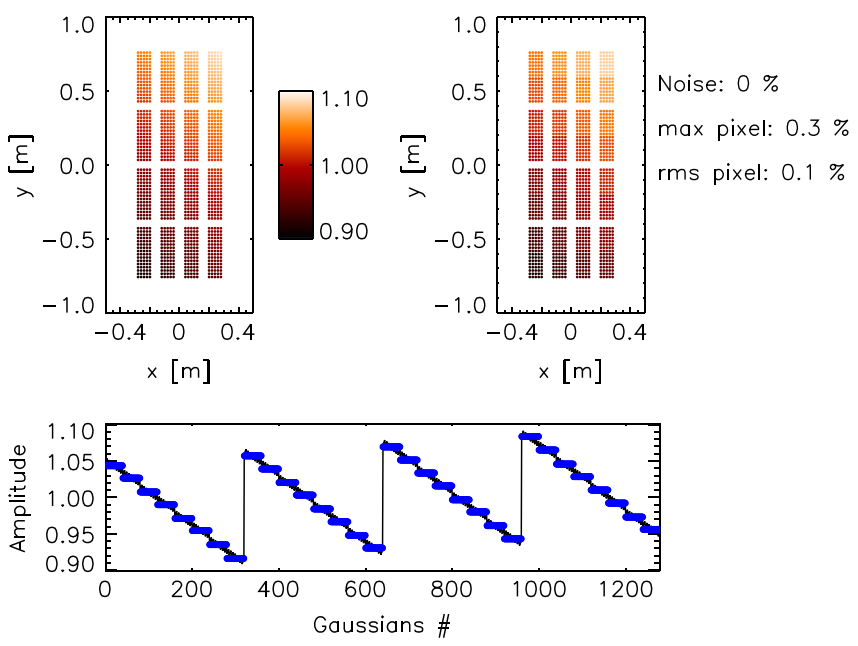

FIG. 6. Phantom 8 (top left) and its tomographic reconstruction (top right) using the 32 pixel configuration. In the bottom panel there are the amplitudes of the simulated Gaussians (the phantom) in black and the amplitudes of the reconstructed Gaussians (blue dots). On the right of the figure there is the value of the noise in percentage added to the line-integrated signals (no added noise in this particular case), the maximum error of the pixel (max pixel), and the rms error of the pixel (rms pixel) in the reconstruction (see the text for the definition of the different errors).

In Fig. 6 the result of the tomographic reconstruction made using the SART method for this phantom is shown. In the top left panel of the figure there is the phantom, with the visible nonuniformity in the two directions. Using the phantom, the line-integrated signals are evaluated $\left(I_{j}\right)$, using Eq. (5), and in this particular case no noise has been added. Then, by applying the SART method [Eq. (12)] the amplitudes of the Gaussians belonging to every pixel have been obtained: the result is shown in the right top panel of Fig. 6 . The color bar refers to both the phantom and its reconstruction. The tomographic reconstruction of the phantom shows its pixel structure, since all the Gaussians inside the same pixel have the same amplitude. In the bottom panel the comparison of the amplitudes of the 1280 Gaussians $\left[A_{g}\right.$ of Eq. (3)] for the phantom (black line) and for the reconstruction (blue dots) is shown. Again, the reconstruction is clearly discrete. The numbers reported on the right-hand side of the figure are the value of the noise (\%) added to the line-integrated signals before applying the tomographic reconstruction (noise, here $0 \%$ ), and the errors in the reconstruction. To evaluate the reconstruction errors, the actual emissivity $\hat{\epsilon}_{i}$ has been defined as

$$
\hat{\boldsymbol{\epsilon}}_{i}=\frac{\int_{i} G_{g} d x d y}{2 \pi \sigma^{2} N_{\text {Gaussians }}},
$$

where the integral $\int_{i}$ means an integral inside the $i$ th pixel. The denominator is the "equivalent area" of the pixel, i.e. the integral of the $N_{\text {Gaussians }}$ standard Gaussians 
( $\sigma_{x}=\sigma_{y}=3 \mathrm{~mm}$ and $\left.A_{g}=1\right)$ inside the $i$ th pixel. So $\hat{\epsilon}_{i}$ is the amplitude of the $N_{\text {Gaussians }}$ standard Gaussians that should be inside the $i$ th pixel such that the area is equal to $\int_{i} G_{g} d x d y$. Then, by naming $\epsilon_{i}$ the emissivity of the reconstructed pixel, the errors are defined as

$$
\begin{gathered}
\text { rms err }=\operatorname{rms}\left(\frac{\hat{\epsilon}_{i}-\epsilon_{i}}{\hat{\epsilon}_{i}}\right) \\
\max \text { err }=\max \left(\left|\frac{\hat{\epsilon}_{i}-\epsilon_{i}}{\hat{\epsilon}_{i}}\right|\right) .
\end{gathered}
$$

The rms error is a measurement of the error of the whole reconstruction, since all pixels are used in its definition; instead the maximum error is a measurement of the worst reconstructed pixel. These two errors are used to estimate the reliability of the reconstruction. In this particular case (phantom 8), when no noise is added to the line-integrated signal, the rms error is $0.1 \%$ and the maximum pixel error is $0.3 \%$. It is important to notice that a certain level of error must be observed, because it is not possible to reconstruct exactly this phantom: in fact, every Gaussian inside a pixel has a different amplitude, whereas in the reconstruction the finest spatials resolution is the dimension of the rectangular pixel. The same phantom is reconstructed also for different noise level added to the line-integrated signals, from 0 to $20 \%$ (rms of the noise to signal ratio, with a uniform distribution). The result of the inversion of this phantom for an intermediate noise level (12\%) is shown in Fig. 7. The tomographic algorithm developed here allows a good reconstruction of this phantom also with noisy data, and the errors in the reconstruction are acceptable. The dependence of the errors in the reconstruction as a function of the noise level is reported in Fig. 8: when increasing the noise, also the errors increase with a linear trend. This is the signature of the dependence of the tomographic algorithm to large- $k$ spatial patterns (small details), which is to
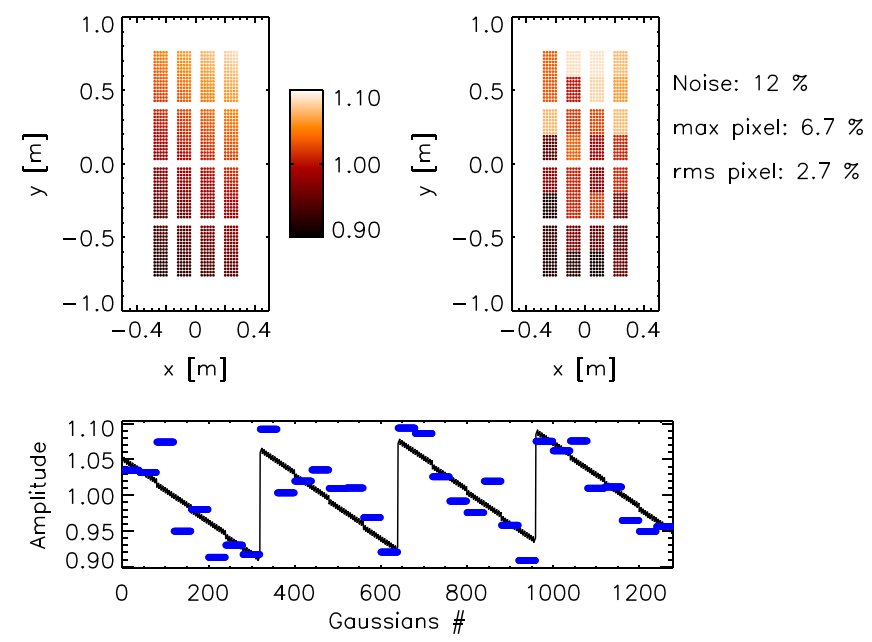

FIG. 7. Phantom 8 (top left) and its tomographic reconstruction (top right) using the 32 pixel configuration with noisy data. The rms of the ratio noise/signal is $12 \%$.

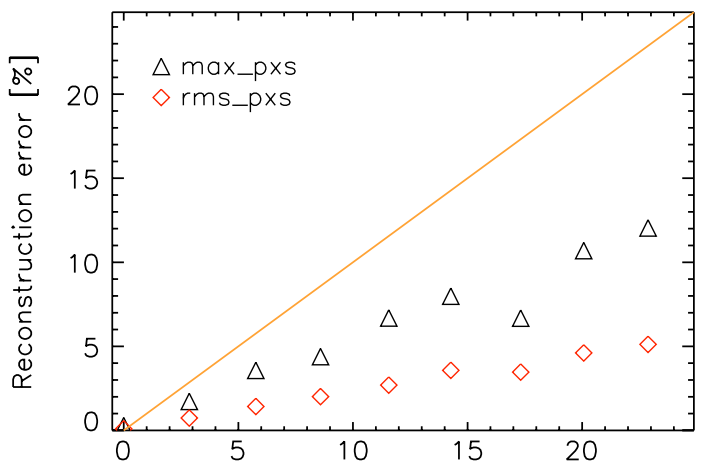

FIG. 8. Error of the tomographic reconstruction of the phantom 8 for the 32 pixel configuration as a function of the noise level added to the line integrated signals: maximum error (black) and rms error (red) as defined in Eq. (14). The line is the reference line $x=y$.

be expected from an unconstrained inversion scheme such as that expressed by Eq. (12). This high- $k$ dependence can be alleviated through a suitable regularization process, which is built in in the SVD method (cutoff of large $k$ ), and is a work in progress for the SART.

A clear result is that both the rms and the maximum errors are always smaller than or comparable to the introduced noise: this is not surprising, since there are more LoSs than pixels (the system of equations used for the reconstruction is over determined) and there is no correlation between the noise introduced in the different LoSs.

To evaluate the level of nonuniformity along the two directions ( $\delta_{x}$ and $\delta_{y}$ ) of the reconstructions, a 2D linear fit of the emissivities of the pixels is computed and compared with the input ones. The result, i.e. the value of the two parameters $\delta_{x}$ and $\delta_{y}$ with their errors is reported in Fig. 9 as a function of the noise added in the line-integrated signals, and compared to the input value (14\% along $y$

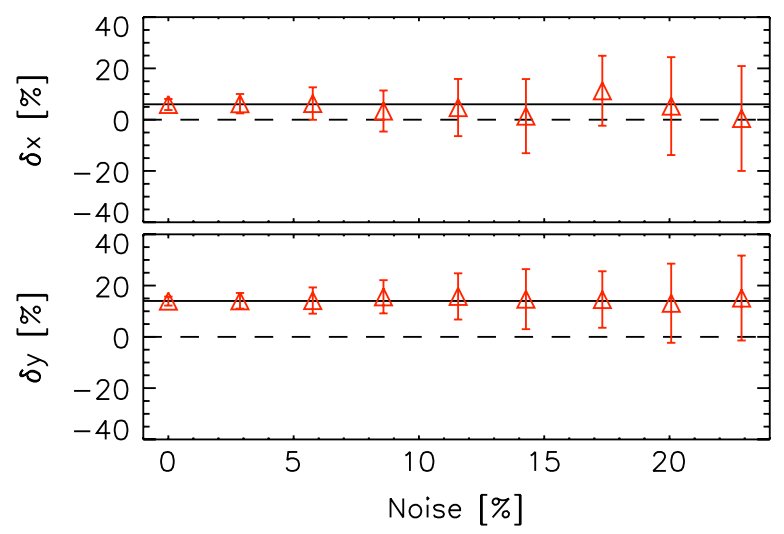

FIG. 9. Uniformity along the two directions of the reconstructed phantom 8 as a function of the noise added to the line-integrated signals. The dotted line corresponds to complete uniformity along that direction, while the continuous line corresponds to the level of nonuniformity of the simulated phantom. 
and $6 \%$ along $x$ ). The nonuniformity of $14 \%$ simulated along the $y$ direction can be correctly determined. The evaluated parameter $\delta_{y}$ is different from 0 (within the error bars) up to a noise level of $20 \%$. The detection of the $6 \%$ nonuniformity along the $x$ direction is more difficult to be discriminated from the 0 value, i.e. from a phantom which is uniform along $x$. Within the error bars, $\delta x$ is different from 0 up to about $5 \%$ of added noise. This means that it is possible to determine that the phantom 8 is not uniform, along $x(6 \%)$ when the noise level of the signals is less than about $5 \%$ and along $y(14 \%)$ when the noise level of the signals is less than about $20 \%$.

\section{B. Uniform phantom}

Phantom 12 is the uniform phantom, which represents the ideal beam. All the Gaussians (beamlets) have the same amplitude, they are centered in the nominal position and their widths along $x$ and $y$ directions are $3 \mathrm{~mm}$. The inversion is reported in Fig. 10 with $12 \%$ added noise. When no noise is added (not shown), the inversion is exact, i.e. both the rms error and the maximum error are 0 . The simulated Gaussians and the reconstructed ones have all the same amplitude: the spatial discretization introduced with the pixels is enough to resolve exactly the phantom, since it is uniform. When noise is added, the reconstruction is no longer exact, but some errors are introduced. Computing the 2D linear fit of the reconstructed emissivity, the correct values $\delta_{x}=\delta_{y}=0$ are obtained (within the error bars) for all noise levels considered (from 0 up to 20\%): the uniform phantom is correctly recognized as uniform.

\section{Criss-cross}

This phantom reproduces a criss-cross of $5 \mathrm{~mm}$ in the beamlets. Horizontal lines of beamlets are shifted
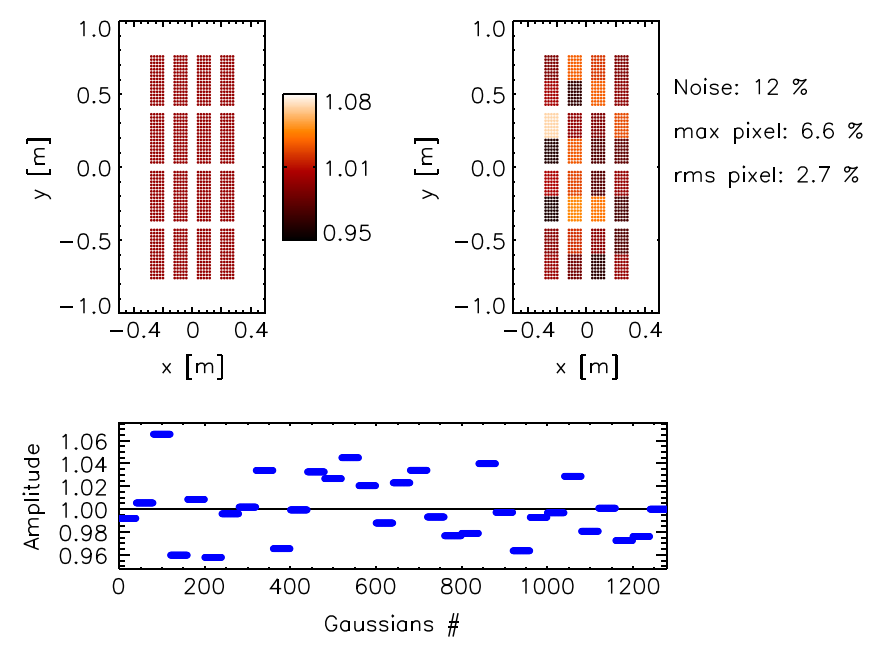

FIG. 10. Phantom 12 (top left) and its tomographic reconstruction (top right) using the 32 pixel configuration with noisy data. The rms of the ratio noise/signal is $12 \%$.

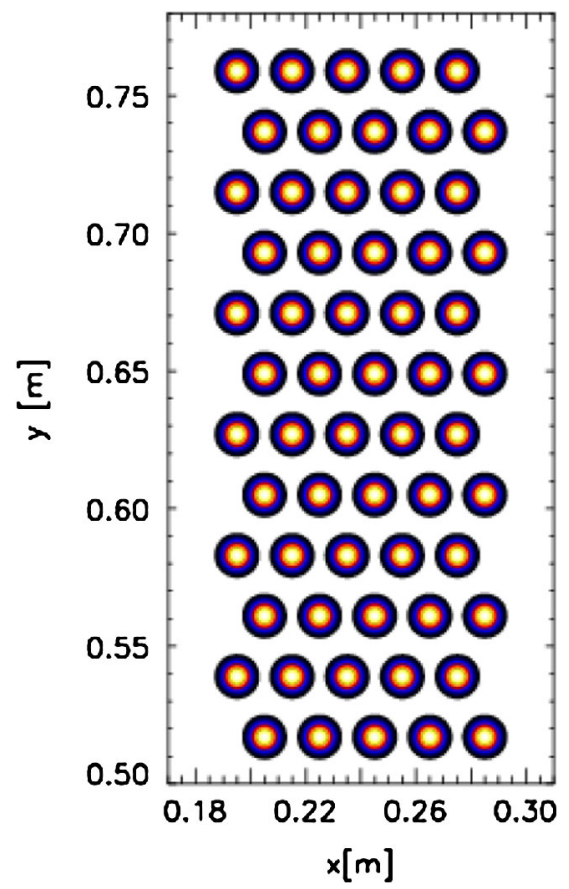

FIG. 11. Zoom of one beamlet group of the phantom with the criss-cross. Each horizontal line of beamlets is shifted alternatively by $+5 \mathrm{~mm}$ and $-5 \mathrm{~mm}$.

alternatively by $+5 \mathrm{~mm}$ and $-5 \mathrm{~mm}$ (see Fig. 11). This is a common phenomenon that affects beams extracted from negative sources [14]. Since the displacements of the beamlets are small compared to their widths, and since the displacement is "symmetric" (i.e. there are some beamlets moving to the left and some other to the right along the $x$ direction), this phantom is easily reconstructed (see Fig. 12), and it is correctly found to be uniform: $\delta_{x}=$ $\delta_{y}=0$ for all the noise levels considered. Because of the
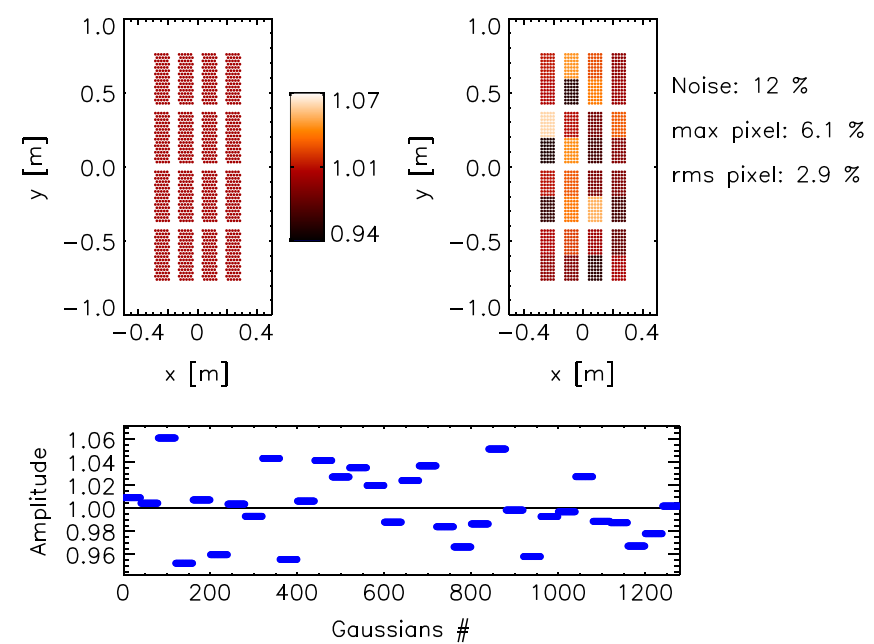

FIG. 12. Phantom 20 (top left) and its tomographic reconstruction (top right) using the 32 pixel configuration with noisy data. The rms of the ratio noise/signal is $12 \%$. 
TABLE I. Spatial shifts along the $x$ and the $y$ direction of the vertices of the 15 fans used for the analyses of the sensitivity of the tomographic algorithm.

\begin{tabular}{|c|c|c|c|c|c|c|c|c|c|c|c|c|c|c|c|}
\hline New fan & 1 & 2 & 3 & 4 & 5 & 6 & 7 & 8 & 9 & 10 & 11 & 12 & 13 & 14 & 15 \\
\hline$\Delta x[\mathrm{~mm}]$ & 0 & 0 & 0 & +10 & -10 & 0 & +10 & 0 & +10 & -10 & +10 & 0 & +10 & 0 & 0 \\
\hline$\Delta y[\mathrm{~mm}]$ & 0 & 0 & 0 & +10 & +10 & +10 & +10 & +10 & -10 & 0 & +10 & +10 & -10 & +10 & 0 \\
\hline
\end{tabular}

finite spatial resolution of the inversion scheme used, it is not possible to reconstruct the position of the beamlets, since in the inversion their positions $\left(x_{0 g}, y_{0 g}\right)$ are fixed. However, the algorithm does not fail, since it converges and the inverted phantom is uniform as the simulated one.

\section{Sensitivity analysis}

In this paragraph the behavior of the tomographic reconstruction with a wrong alignment of the optics is studied. This aspect is of particular importance, since the real LoSs cannot be aligned with infinite precision and so they are not exactly the lines of sight used in the inversion algorithm. This means that the LoS geometry used in the algorithm is not exactly the real one. To estimate the sensitivity to this discrepancy, the line-integrated signals of phantom 8 are evaluated with a set of LoSs which is slightly different from that used for the tomographic reconstruction. To modify the geometry of the lines of sight, 11 fans randomly chosen are shifted arbitrarily by $+10 \mathrm{~mm}$ or $-10 \mathrm{~mm}$ along $x$ and/or $y$ directions. The shifted fans with their displacements are reported in Table I and they represent a very extreme condition since the alignments can be easily performed with higher accuracy $( \pm 1 \mathrm{~mm})$. In Fig. 13 the rms error of the reconstruction made with the misaligned fans is compared with the same error for the correct fan positions. The first and most important result to underline is that, even with a misalignment of the lines of sight, the algorithm can invert the phantom, and the errors of the reconstruction are acceptable. Using different positions of the LoS for the

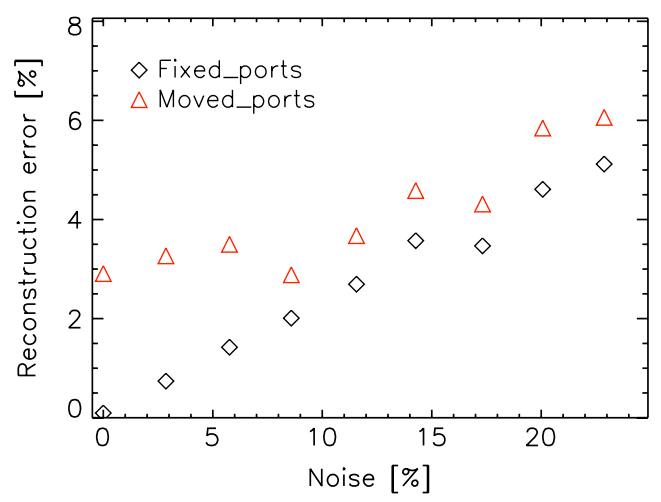

FIG. 13. Comparison of the rms error of the tomographic reconstruction as a function of the added noise level for the correct position of the fans and with the fans moved. line-integrated signals and for their inversion, causes an increase of the reconstruction error. In addition, a fixed error level appears at low noise level: about $3 \%$ for the 32 pixel configuration, showing that the error introduced by the bad alignment of the optics dominates here over the added noise to the LoS signals. The main result of this simulation is that, with this geometry of the LoSs, the algorithm is not too sensitive to a realistic misalignment of the optics.

\section{CONCLUSIONS}

The tomographic system developed for a beam from a negative ion source has been described; an inversion algorithm has been implemented and tested for different beam configurations in SPIDER in order to demonstrate that the diagnostic will be capable of characterizing the beam uniformity. Different phantoms have been inverted with the developed tomographic algorithm, studying also the effect of the noise in the reconstruction accuracy. The nonuniform phantom is correctly reconstructed and the nonuniformity along both $x$ and $y$ directions is correctly evaluated. In particular, it has been stated that the nonuniformity along $x(6 \%)$ is correctly determined when the noise of the signal is less than about 5\% and the nonuniformity along $y(14 \%)$ is correctly determined when the noise of the signal is less than about $20 \%$. Therefore the highest noise level of the line-integrated signals above which the tomographic inversion begins to fail can be considered 10\% (rms noise). However, for commercial CCDs that could be used as light detectors, the expected noise is lower. To obtain these results, 3127 lines of sight grouped into 15 cameras (projections) are used, and their configuration has been optimized in order to provide a good coverage of the whole beam area. The developed algorithm is based on the simultaneous algebraic reconstruction technique (SART), which gives better reconstruction than the SVD technique when noisy data are used. To be able to resolve also misalignments of the beamlets which are comparable to their widths, the algorithm developed could be improved by adding more free parameters, like the position of the centers of each beamlet $\left(x_{0}, y_{0}\right)$. This aspect will be the subject of further studies. The second improvement of the algorithm could be the addition of a regularization scheme [25-27] to avoid small spatial scale fluctuations in the 2D image and to increase of the spatial resolution of the reconstruction, by increasing the number of the pixels from 32 to 64 . 


\section{ACKNOWLEDGMENTS}

The authors gratefully acknowledge Alberto Alfier, Luca Lotto, and Chiara Piron for the experimental work in designing and testing the optics and the CCD camera, and Barbara Zaniol for useful comments and discussions about the spectroscopy of the beam. This work was set up with collaboration and financial support of Fusion for Energy.

[1] P. Sonato et al., Fusion Eng. Des. 84, 269 (2009).

[2] U. Fantz et al., Nucl. Fusion 46, S297 (2006).

[3] U. Fantz et al., Nucl. Fusion 49, 125007 (2009).

[4] C. F. Burrell, W. S. Cooper, R. R. Smith, and W. F. Steele, Rev. Sci. Instrum. 51, 1451 (1980).

[5] P.A. van der Elsen, E. J.D. Pol, and M. A. Viergever, IEEE Eng. Med. Biol. Mag. 12, 26 (1993).

[6] E. Speth et al., Nucl. Fusion 46, S220 (2006).

[7] L. R. Grisham et al., Nucl. Fusion 41, 597 (2001).

[8] O. Kaneko et al., Nucl. Fusion 43, 692 (2003).

[9] L. R. Grisham, IEEE Trans. Plasma Sci. 33, 1814 (2005).

[10] G. A. Cottrell, J. Phys. E 15, 432 (1982).

[11] G. A. Cottrell, Rev. Sci. Instrum. 55, 1401 (1984).

[12] S. Yue-Jiang et al., Chin. Phys. Lett. 24, 1629 (2007).
[13] S. W. Lee et al., Nucl. Instrum. Methods Phys. Res., Sect. A 422, 693 (1999).

[14] Y. Okumura et al., in Proceedings of the 18th International Conference on Fusion Energy, Sorrento, Italy (2000).

[15] N. Umeda et al., Nucl. Fusion 43, 522 (2003).

[16] K. Tsumori et al., Rev. Sci. Instrum. 81, $02 B 117$ (2010).

[17] F. Natterer, The Mathematics of Computerized Tomography (Teubner, Stuttgart, 1986).

[18] A.C. Kak and M. Slaney, Principle of Computerized Tomography Imaging (IEEE Press, New York, 1988) [http://cobweb.ecn.purdue.edu/malcolm/pct/pct-toc.html].

[19] R. Pasqualotto et al., Nucl. Instrum. Methods Phys. Res., Sect. A 623, 794 (2010).

[20] P. Holmes, J. L. Lumly, and G. Berkooz, Turbulence, Coherent Structures, Dynamical Systems and Symmetry (Cambridge University Press, Cambridge, England, 1996).

[21] D. Verhoeven, Appl. Opt. 32, 3736 (1993).

[22] P. M. Subbarao, P. Munishi, and K. Muralidhar, NDT\&E International 30, 359 (1997).

[23] H. S. Ko and K. D. Kim, Exp. Fluids 27, 542 (1999).

[24] A. H. Andersen and A.C. Kak, Ultrasonic Imaging 6, 81 (1984).

[25] A. N. Tikhonov, Sov. Math. Dokl. 4, 1035 (1963).

[26] P. C. Hansen, SIAM Rev. 34, 561 (1992).

[27] P.C. Hansen and D. P. O'Leary, SIAM J. Sci. Statist. Comput. 14, 1487 (1993). 